\title{
Sequential use of topical imiquimod and photodynamic therapy in Extrammamary Paget's disease: a case report and review of the literature.
}

\author{
LM Nieto-Benito ${ }^{1}$, V Parra-Blanco ${ }^{1}$, RM Suárez-Fernandez ${ }^{1}$, and L Barchino-Ortiz ${ }^{1}$ \\ ${ }^{1}$ Affiliation not available
}

May 27, 2020

"TWEETABLE" ABSTRACT:

Extramammary Paget's treatment with imiquimod and photodynamic therapy in combination can be easily tolerated and used safely and effectively.

\section{KEY WORDS:}

Carcinoma in situ; Imiquimod; Paget's disease, Extrammamary; Photochemotherapy; Photodynamic Therapy.

\section{KEY POINTS:}

- EMPD represents an intraepithelial carcinoma whose diagnosis is often delayed and it is based on histopathological and Immunohistochemistry. Once diagnosis is made, investigation for an underlying carcinoma has to be done.

- Extramammary Paget's treatment is a difficult issue due to intolerance and recurrence; topical treatments can be an alternative and imiquimod and photodynamic therapy can be used safely and effectively in combination.

Extramammary Paget's disease (EMPD) represents an intraepithelial carcinoma which occurs in apocrine gland-bearing skin ${ }^{1}$. Surgery is the treatment of choice but non-invasive options such as radiotherapy or topical chemotherapy are alternatives to consider in patients that are medically unfit for surgery or recur ${ }^{2}$.

An 83-year old woman presented with a 2-year history of pruritus on her vulvar area. She had consulted several physicians who had prescribed antifungal and corticosteroid ointments without improvement. The lesion had been slowly growing and had started to be painful and to present small erosions on its surface. Physical examination revealed erythematous and scaly plaques with superficial small erosions affecting the skin of her right labia majora and gluteus (Fig.1).

Due to failure to treatment, punch biopsy was performed (Fig.2). An epidermal infiltration by large atypical, eosinophilic cells, with wide cytoplasm and prominent marginal nuclei, scattered throughout all layers of epidermis was found. This proliferation was not found deeper in dermis nor subcutaneous tissue. Immunohistochemistry showed positivity for cytokeratin 7 (CK7) and CK20 stain was negative. These histopathological findings were consistent with Paget cells, and, suggestive of primary EMPD. Nevertheless, body computed tomography, gastroscopy, colonoscopy, gynaecologic ultrasonography and cervical cytology were performed without evidence of malignancy.

Surgery was offered but patient preferred a conservative option. Imiquimod 5\% cream was started: 3 applications per week, once at night, during 16 weeks. Due to multifocal lesions, treatment was performed first in 
the vulva and, secondly, in the gluteus. Complete response was obtained clinically and histopathologically.

Recurrence was experienced six months after and imiquimod was restarted. This second application was not tolerated and photodynamic therapy (PDT) using methyl aminolevulinate was initiated. Complete remission was achieved after four sessions of PDT (red light, $630 \mathrm{~nm}, 37 \mathrm{~J} / \mathrm{cm} 2,7$ minutes; two sessions per area, one session per week). After twelve months, imiquimod was successfully reused with control of disease after second recurrence.

Extramammary Paget's disease (EMPD) was first described in 1889 by Croker as a cutaneous carcinoma located in the genital area of a 60 year-old man that presented with clinical and histological features similar to those found in Mammary Paget's disease ${ }^{2}$, defined by James Paget in 1874. Although its pathogenesis remains controversial, in primary disease, it represents an intraepithelial carcinoma most likely derived from the intraepithelial sweat duct ${ }^{2}$.

Extramammary Paget's disease (EMPD) commonly involves the vulva but can also be found in perianal skin, scrotum, penis and axilla. Pruritus is the most common symptom; burning, irritation, pain, tenderness, bleeding and swelling can also be present ${ }^{3}$. Patients develop well-defined erythematous and scaly plaques that can associate crust, liquenification, erosions and ulceration. Its characteristic "cake-icing" appearance was first described by Dubreuilh ${ }^{4}$, due to the presence of white islands and bridges of hyperkeratotic epithelium upon erythema.

It can be easily misdiagnosed as a benign dermatitis and be mistaken for eczema, psoriasis, fungal infection and seborrheic dermatitis ${ }^{2}$. That is the reason why diagnosis and definitive treatment are often delayed. Skin biopsies should be performed in all patients with eczematous or infectious lesions that have failed to respond to standart topical treatment ${ }^{3}$.If lymphadenopathies or palpable nodules are present, they raise suspicion for invasive disease ${ }^{2}$.

EMPD's diagnosis rests on histological criteria. Immunohistochemistry studies are mandatory to make the diagnosis and to exclude anogenital intraepithelial neoplasm and malignant melanoma ${ }^{5}$. EMPD can also represent an epidermotropic metastasis from a distant malignant neoplasm (secondary EMPD); this technique also contributes to differentiate between primary and secondary EMPD ${ }^{6}$.

Paget's cell stain for markers of apocrine and eccrine lineage such as low molecular weight cytokeratins (such as CK7), gross cystic disease fluid protein (GCDFP-15), periodic acid-Schiff (PAS) and carcinoembryonic antigen $(\mathrm{CEA})^{5-6}$ and, therefore, this technique helps to identify the likely cell of origin as primary and secondary disease show different immunophenotypes ${ }^{7}$. Cytokeratin 20 (CK20) is an epithelial marker with restricted expression compared to $\mathrm{CK}^{6}$.

Primary EMPD stains for CK7 and GCDFP-15, being CK20 negative; secondary EMPD is CK20 positive and GCDFP-15 negative ${ }^{7}$. Our case showed an Immunohistochemistry profile of primary disease (CK7 positive; CK 20 negative) (Fig 2). After the diagnosis of EMPD is made, especially with a secondary disease profile, investigation for an underlying carcinoma has to be made. Gastrointestinal and genitourinary neoplasm can be found in up to $20 \%$ of EMPD patients ${ }^{2-3}$.

Although generally accepted as the standard treatment, all surgical modalities are associated with high local recurrence rates ${ }^{8}$. Margins are difficult to assess even in frozen sections and wide local excision have been associated with important recurrences rates as well and conservative techniques are been performed nowadays. Radiotherapy can be an alternative as primary treatment but, it should be only consider in patients medically unfit for surgery or after recurrence ${ }^{9}$.

Topical chemotherapy agents ${ }^{9-10}$, such as 5-fluorouracil, imiquimod and bleomycin and photodynamic therapy, have been used to treat EMPD. They can be useful as cytoreduction prior to surgery, early disease recurrence and as primary treatment for those where surgery is not an option. All topical modalities can produce severe local reactions and a subsequent lack of compliance of treatment. There are studies that have demonstrated the efficacy ${ }^{10}$ and tolerability of topical treatments with a neoadyuvant ${ }^{8}$ or sequential/combined use ${ }^{9}$. Sequential use of topical chemotherapies (imiquimod and PDT) can be considered 
an effective alternative that can contribute to tolerate treatment schedules, reduce recurrences and favour control disease.

\section{DISCLOSURE OF INTEREST}

No relevant financial, personal, political, intelectual or religious interest to declare.

\section{CONTRIBUTION TO AUTHORSHIP}

All authors have participated sufficiently in the work to take public responsability for the content, including participation in the concept, design, anaylisis, writing and approval of the final version of the manuscript.

Each author certifies that this material has not been and will not be submitted to or published in any other publication before its appearance in the BJOG.

\section{PATIENT'S CONSENT}

Patient consent to the author's and, therefore, to the journal this work was subscribed in, collecting, using and disclosing the information for the purpose. The patient read and had the opportunity to review the article before signing and submitting. This article was authorized prior to publication by Hospital General Universitario Gregorio Marañón CEIC (Ethics Committee).

\section{FUNDING}

The authors have not received any financial support or funding on this project. The authors have no significant interest with commercial supporters.

\section{REFERENCES}

1. Mehrtens SH, Tharakaram S. Extramammary Paget's Disease. N Engl J Med 2017;376:35. Doi: 10.1056/NEJMicm1610755.

2. Zaleski M, Ferringer T. Extramammary Paget's Disease. Cutis 2015;95:315-6.

3. Choi J, Zemek A, Lee GK, Kin C. Perianal Extramammary Paget's Disease: More than Meets the Eye. Dig Dis Sci 2018;63:2853-7. Doi: 10.1007/s10620-018-5089-1.

4. Dubreuilh W. Paget's disease of the vulva. Br J Dermatol 1901;13:407-13.

5. Nogano T, Kotani S, Omori M, Kosaka H, Ogawa M. Pigmented extramammary Paget's disease: Pitfalls of diagnosis. J Dermatol 2017;44:1323-5. Doi: 10.1111/1346-8138.13684.

6. Fernandez-Flores A, Eraña I, Cuevas J. "Extramammary-Type" Paget Disease of the breast. Am J Dermatopathol 2018;40:711-20.

7. St Claire K, Hoover A, Ashack K, Khachemoune A. Extramammary Paget Disease. Dermatol Online J $2019 ; 25$.

8. Toledo F, Silvestre JF, Cuesta L et al . Sequential use with imiquimod and surgery in Extramammary Paget's Disease. Dermatol Ther 2012;25:81-5. Doi: 10.1111/j.1529-8019.2012.01428.x

9. Apalla Z, Lallas A, Tsorova A et al . Complete response of Extramammary Paget's Disease With Imiquimod and PDT: report of two cases. Photodermatol Photoimmunol Photomed 2018;34:273-5. Doi: 10.1111/phpp.12386.

10. Rioli DI, Samimi M, Beneton N et al . Efficacy and tolerance of photodynamic therapy for vulvar Paget's disease: a multicentric retrospective study. Eur J Dermatol 2018;28:351-5. Doi: 10.1684/ejd.2018.3289

FIGURE LEGENDS 
Figure 1 a) Clinical image at presentation: erythematosquamous plaques affecting the skin of the right labia majora and ipsilateral gluteus. b) Clinical detail: Superficial erosions on the surface of the plaques separate white hiperkeratotic areas giving the characteristic "cake-icing" or "strawberries and cream" appearance.

Figure 2 . Histopathology a) Punch skin biopsy - Hematoxylin and eosin (10x): epidermal infiltration by large atypical cells with eosinophilic, wide cytoplasm and prominent marginal nuclei, consistent with Paget's cells, in an acanthotic epidermis. b) Punch skin biopsy - Hematoxylin and eosin (20x): Paget's cells scattered throughout all layers of epidermis, arranged predominantly in groups. c) Immunohistochemistry CK7 stain: positive in skin Paget's cells. d) Immunohistochemistry CK20 stain: negative in skin Paget's cells.

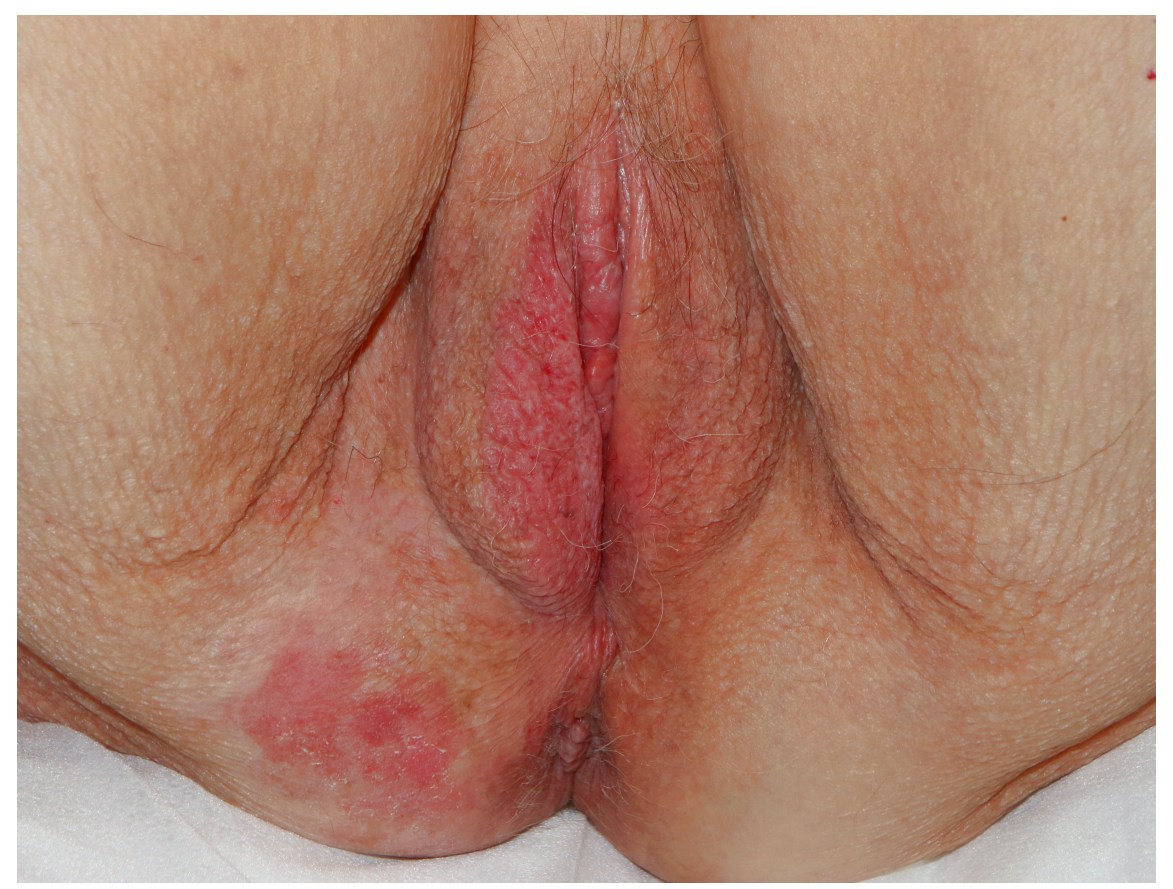



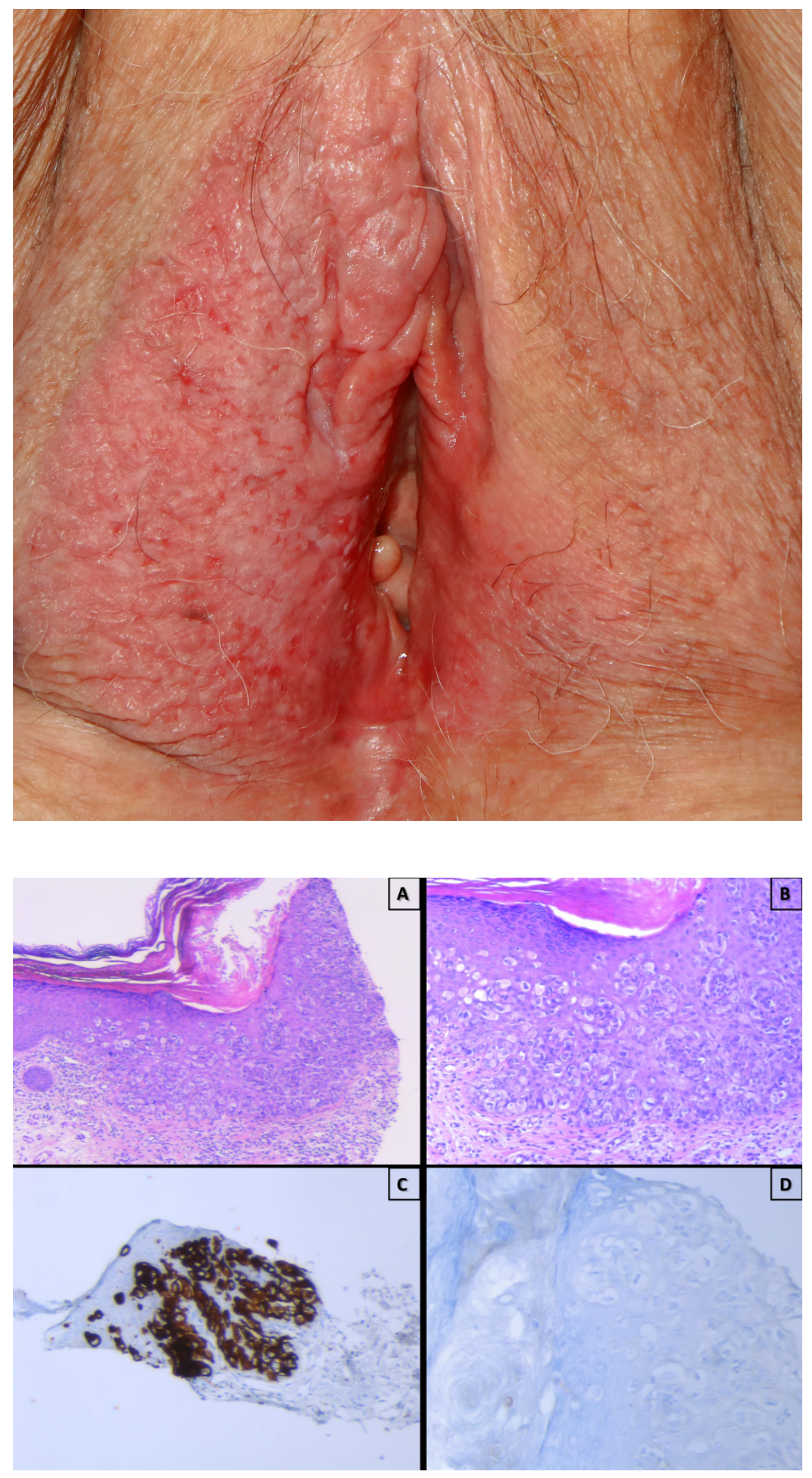